\title{
TOLERÂNCIA AO COBRE EM ECÓTIPOS DE Schinus lentiscifolius March (ANACARDIACEAE) DE ÁREAS DE MINERAÇĀO NO RIO GRANDE DO SUL,' BRASIL
}

\author{
Maria Luiza Porto
}

Recebido em 28-01-89. Aceito em 08-11-98.

RESUMO - Sementes de Schinus lentiscifolius March, provenientes de duas áreas de mineração do Rio Grande do Sul, foram submetidas a testes de exposição ao $\mathrm{Cu}$ $\mathrm{SO}_{4}$, visando comprovar a tolerância ao cobre na germinação e no desenvolvimento de plântulas. Os testes demonstraram que os dois clones reagem diferentemente ao cobre, porém ambos apresentaram mecanismos de tolerância. Plântulas provenientes de sementes da área de maior concentração de cobre no solo (minas do Seival) apresentam sintomas de toxidez mais tardios do que plântulas provenientes de sementes da área de menor concentração deste íon (mina Sanga Negra).

Palavras-chave: tolerância a cobre, Schinus lentiscifolius, áreas de mineração.

\begin{abstract}
ABASTRACT - Seeds from Schinus lentiscifolius March collected in two mining areas in the state of Rio Grande do Sul, Brazil, were submitted to CuSO 4 exposure tests to confirm copper tolerance during germination and plantule development. The results showed that the two distinct clones respond differently to copper, but both presented tolerance mechanisms. Plantules originated from seeds collected in the area with higher soil copper concentration (minas do Seival) presented toxicity symptoms later as compared to those plantules originated from seeds collected in the area with lower $\mathrm{CU}^{2}+$ concentration (mina Sanga Negra).
\end{abstract}

Key Words: Copper toleleance, Schinuslentiscifolius, mining areas.

1 - Parte da tese de doutorado desenvolvida na Universidade de Ulm, República Federal da Alemanha. 2 - Universidade Federal do Rio Grande do Sul. Departamento de Botânica e Centro de Ecologia do Instituto de Biociências. Avenida Paulo Gama s/n, 90049, Porto Alegre, RS. Brasil Pesquisadora 2A do CNPq. Processo no 305062/76. 


\section{Introdução}

As áreas de mineração de minas do Seival e minas de Sanga Negra, objeto de nossos estudos no Rio Grande do Sul, caracterizam-se pela presença de minérios de cobre, wolfrâmio e estanho. Atualmente, Seival está sendo reativada para exploração de ouro, e Sanga Negra não se encontra mais em atividade. Outros metais, associados ao cobre acham-se sob a forma de sulfetos, ao passo que os associados ao wolfrâmio estão sob a forma de óxidos.

Nos solos destas áreas, Porto $(1981,1986)$ constatou quantidades acima da normalidade de cobre, chumbo, molibdênio, níquel, prata e quantidade-traço de cádmio. Além destes fons metálicos, também ê citada, por Leinz e Pinagel (1945), a ocorrência de wolfrâmic e estanho em Sanga Negra. Em uma quantificação da disponibilidade de fons para as plantas, Porto (1981) obteve até 10 ppm de cobre em locais em Sanga Negra, enquanto que, em Seival, constatou ate $9900 \mathrm{ppm}$ deste mesmo elemento em amostra diretamente sobre a estrutura mineralizada usando o método de extração com solução 0,1 normal de $\mathrm{HNO}^{3}$. Tais valores, principalmente os obtidos em Seival,caracterizam solos metalíferos, que podem ser próprios para exploração mineral (Ernst, 1974).

Plantas que crescem sobre solos metalíferos apresentam, muitas vezes, modificações morfológicas e anatômicas, além de alterações fisiologicas (Porto 1981, 1986). Estas modificações levam a uma alteração na vegetação, determinando o que se denomina savana arbustiva metalofila - Schwermetallvegetation, Strauchsavanne (Ernst 1974, Porto 1981, 1986a).

Schinus lentiscifolius March, uma espécie de aroeira, é a espécie arbustiva mais freqüente nas savanas metalofilas no Rio Grande do Sul, acumulando altas concentrações de metais pesados em seus tecidos, como quantidades anormais de ate 205 ppm de cobre, 32 ppm de molibdênio, 17 ppm de níquel a 10 ppm de chumbo nas folhas, além de quantidades não significativas de prata, cádmio e estanho (Porto, 1986).

Espécies como $S$. lentiscifolius podem, segundo Antonovics et al. (1971), apresentar mecanismos de tolerância ao excesso de metais pesados. Ao mesmo tempo, as condições dos ambientes metalófilos levam a uma seleção dos indivf́duos, podendo a vegetação destes locais apresentar endemismos: novas espécies, formas ou variedades ou, simplesmente, ecótipos que se encontram em processo de especiação, agindo os metais como um fator de seleção sobre o potencial gênico de uma população.

A tolerância de plantas ao excesso de metais pesados pode se dar de diferentes maneiras, seja através da regulação na concentração destes em seus tecidos, pela ação metabólica, seja através de outros mecanismos, que se manifestarão em aspectos químicos, físicos ou fisiológicos. Ernst (1974), Coughtrey \& Martin (1978), Hougan et al. (1977a), Hougan et al. (1977b), Howard-William (1971), Wainright \& Woolhouse (1977) e Karataglis (1978) verificaram diferentes tipos de mecanismos de tolerância ao excesso de metais pesados em clones de plantas de áreas de mineração na Europa, América do Norte e África. 


\section{Material e Métodos}

Testes de tolerância podem ser realizados pela verificação da germinação e do controle do desenvolvimento das plantas filhas.

Sementes de Schinus lentiscifolius March - aroeira - provenientes das áreas de mineração das minas do Seival, no município de Lavras do Sul, e da mina Sanga Negra, no município de Encruzilhada do Sul, foram coletadas e colocadas para germinar em sete placas de Petri. Em cada uma destas, foram colocadas 25 sementes, realizando-se regas periódicas com soluções molares (Mol/1) de $10^{-1}, 10^{-2}, 10^{-3}, 10^{-4}, 10^{-5}$ e $10^{-6}$ de sulfato de cobre (CuSO 4$)$, com exceção do controle, que recebeu apenas água bidestilada.

As placas foram acondicionadas em casa de vegetação com umidade e temperatura controladas. Durante $\mathbf{3 0}$ dias, observou-se o experimento, anotando-se o número de sementes germinadas por placa, e, em seguida, calculou-se o percentual de germinação em relação à capacidade de germinação de cada lote (avaliada pelo controle).

Sementes provenientes destas mesmas plantas foram postas a germinar em terra para cultivo, e, após três meses, as plântulas foram transplantadas para culturas hidropônicas, com solução nutritiva de Knop, que continham concentrações de $2 \times 10^{-1}, 10^{-2}, 10^{-3}, 10^{-4}, 10^{-5}, 10^{-6} \mathrm{Mol} / \mathrm{l}$ de sulfato de cobre $\left(\mathrm{CuSO}^{4}\right)$. No controle, foi usada somente a solução de Knop. Estas culturas tamberm foram acondicionadas em casa de vegetação. Após quatro meses, determinou-se a quantidade de cobre acumulada nas folhas dessas plantas. A determinação foi feita usando-se um espectrofotômetro de absorção atômica (Perkin-Elmer AAS 400) acoplado com forno de grafite (HGA 74) e analisador eletrônico (Perkin-Elmer 420). A digestão do material foi feita segundo Kotz et al. (1972). As características qualitativas do grau de desenvolvimento, presença de cloroses, necroses, e escreções de sal foram anotadas durante o período de exposição destas plantas.

\section{Resultados}

Constatou-se que as sementes procedentes de Seival apresentaram uma capacidade de germinação de $36 \%$ e as de Sanga Negra, de $56 \%$. Estes valores foram considerados para o cálculo do percentual de germinação.

Pela análise da percentagem de germinação das sementes submetidas a diferentes concentrações de $\mathrm{CuSO}_{4}$, verifica-se que as procedentes de Seival apresentaram maior resistência, obtendo-se germinação (11\%) até a concentração de $10^{-1} \mathrm{Mol} / 1$ de $\mathrm{CuSO}_{4}$ (tabela 1). Esta percentagem aumenta gradativamente ate atingir, na concentração de $10^{-6} \mathrm{Mol} / 1,111 \%$, valor acima do obtido no controle. Por outro lado, as sementes de Sanga Negra começam a apresentar germinação somente em concentrações inferiores a $10^{-2} \mathrm{Mol} / \mathrm{l}$. Neste concentração as sementes provenientes de Seival já apresentam uma percentagem de germinação de $33,3 \%$. Verifica-se que na concentração de $10^{-3} \mathrm{Mol} / 1$ 
ocorrem $77,7 \%$ de germinação para as sementes de Seival e somente $25 \%$ para as de Sanga Negra.

TABELA 1

Taxa de Germinação relativa (\%/Controle)

$\mathrm{CuSO}_{4} \mathrm{Mol} \quad 10^{-1} 10^{-2} 10^{-3} 10^{-4} 10^{-5} \quad 10^{-6} \quad \begin{gathered}\text { Taxa de germinação } \\ \text { do controle (\%) }\end{gathered}$

$\begin{array}{lrllllll}\text { Seival } & 11 \% & 33,3 & 77,7 & 77,7 & 100 & 111 & 36 \\ \text { Sanga Negra } & 0 & 25 & 42 & 50 & 64 & 56\end{array}$

Percentual de germinação em sementes provenientes de ecótipos de duas áreas de mineração do Rio Grande do Sul (minas do Seival e mina Sanga Negra).

Nas plântulas cultivadas em solução de Knop, com concentração crescentes de sulfato de cobre, foi possível constatar, já no primeiro mês de cultivo, uma redução no tamanho das plantas, cloroses e necroses nas folhas, alem de excreções de sal de cobre na superfície destas, nas concentrações de 0,2 e $10^{-1}$ $\mathrm{Mol} / \mathrm{l}$. De uma maneira geral, observou-se que as plantas procedentes de sementes de Sanga Negra apresentaram sintomas de toxidez mais acentuados e mais processos. Após mais dois meses de observação, estes sintomas se acentuaram para ambos os clones, atingindo, tamberm, as plantas cultivadas em solução de $10^{-3} \mathrm{Mol} / 1$. Nestas, porem, os sintomas foram mais atenuados, e a toxidez não chegou a provocar a morte das plantas. Com mais um mês de observação, o comportamento foi quase idêntico ao anterior. Neste período, coletaram-se folhas de ambos os clones das plantas expostas ao cobre em soluções de $10^{-1}$ e $10^{-3} \mathrm{Mol} / \mathrm{l}$, e o conteúdo de metal acumulado foi calculado em relação à matéria seca, obtendo-se, na concentração de $10^{-3} \mathrm{Mol} / 1$, para Seival, uma concentração de 610 ppm de cobre, e, para Sanga Negra, 525 ppm. Em concentrações mais elevadas $\left(10^{-1}, 10^{-1}\right.$ e $\left.2 \times 10^{-1}\right)$ os tecidos vegetais já estavam mortos e os restos foliares repletos de cristais de sulfato de cobre, tornando impossível a determinação deste metal nos tecidos.

\section{Discussão}

A capacidade de uma planta colonizar áreas de mineração requer plasticidade gênica, que se reflete em alterações metabólicas, demonstrando diferentes graus de tolerância expressos por alterações morfológicas e fisiológicas. A adaptação destas plantas decorre do fato de derivarem de plantas com plasticidade de adaptação a ambientes extremos e possuírem mecanismos gênicos que proporcionam a adaptaçã̃o à colonização destas áreas. Schinus lentiscifolius ocorre em solos metalíferos do Rio Grande do Sul, apresentando uma série de 
sintomas de adaptações a estas condições, entre estes, mecanismos de tolerância ao elemento testado, o cobre, o qual encontra-se em abundância nos locais estudados. Ernst (1974) e Baumeister \& Ernst (1978) afirmam que a tolerância das plantas é metalo-específica, quando nos locais existe enriquecimento de um metal. Porém, quando o enriquecimento é de mais de um metal, existe tolerân. cia também a este outro. Em Seival e Sanga Negra, como já foi comprovado em trabalhos de Porto $(1981,1986)$, existe enriquecimento de cobre e de outros íons, como molibdênio, chumbo e níquel e, em menor quantidade, estanho, câdmio e prata, podendo haver tolerância não somente ao cobre, como nosso, experimento demonstra, mas também aos demais metais da área considerada.

Supondo-se que, nas áreas de maior e menor ocorrência de cobre (Seival e Sanga Negra, respectivamente), tenham se formado clones distintos de $S$. lentiscifolius, tolerantes ao cobre, testaram-se suas resistências na germinação. As sementes de Seival apresentaram maior resistência a $\mathrm{CuSO}_{4}$, tendo em vista a ocorrência de germinação desde uma concentração $10^{-6} \mathrm{Mol} / 1$ até $10^{-1}$. As sementes de Sanga Negra, ao contrário, germinaram somente até a concentração de $10^{-3} \mathrm{Mol} / \mathrm{l}$. Comportamentos diferentes, durante a germinação, de sementes submetidas a diferentes concentrações de metais pesados foram verificados por Ernst (1974), Mukherji \& Gupta (1972), Gupta \& Mukherji (1977) e Miles \& Parquer (1979). Em ecótipos de Armeria maritima, foi verificado, por Ernst (1974, 1975), comportamento similar ao obtido na germinação de sementes de Schinus lentiscifolius (tabela 1), onde ficou evidente que as sementes de Seival germinaram em percentagem mais alta do que as de Sanga Negra. Em sementes de Armeria maritima, provenientes de três solos distintos (ricos em cloreto de sódio, em metais pesados e do tipo serpentina), houve reações similares, quando submetidas a $\mathrm{CuSO}_{4}$, germinando em maiores percentagens as provenientes de solos ricos em metais pesdos e as de solos do tipo serpentina (que também concentram muitos metais pesados).

Outro aspecto que pode ser considerado em relação à tolerância ao cobre é que, nas sementes provenientes de Seival, o cobre, em baixa concentração, favoreceu a germinação, pois na concentração de $10^{-6} \mathrm{Mol} / \mathrm{l}$, houve percentagem de germinação acima do controle. Tais resultados encontram similaridade com os obtidos por Horschraft (1961), que evidenciam que Becium homblei, espécie que pode naturalmente suportar altas concentrações de cobre no solo, precisa, para germinar, de 50 a 600 ppm de cobre. Este mesmo autor afirma que esta espécie é endêmica de solos cupríferos de Zimbabwe (antiga Rodésia), onde se comporta como ecótipo fisiológico, o qual requer alta concentração de cobre para um crescimento saudável.

O segundo teste - desenvolvimento das plantas em soluções nutritivas contendo $\mathrm{CuSO}_{4}$ - demonstra que concentrações de $0,2 \mathrm{Mol} / 1$ e $10^{-1} \mathrm{Mol} / 1$ são tóxicas para ambos os clones de plantas. Brooks (1972), classifica o cobre como elemento pertencente ao grupo de metais muito tóxicos, sendo que sintomas de toxidez já podem aparecer quando as concentrações no substrato são menores que $1 \mathrm{ppm}$. Juntamente com o cobre, classificam-se assim o berilo, mercu- 
rio, prata e estanho. Baumeister \& Ernst (1978), de acordo com o que já foi constatado por Hunter \& Vergano (1953) em plantas de aveia, afirmam que os metais podem ser ordenados quanto ao prejuízo causado pelo excesso nas suas concentrações $\mathrm{Ni}<\mathrm{Cu}<\mathrm{Co}<\mathrm{Cr}<\mathrm{Zn}<\mathrm{Mo}<\mathrm{Mn}$. Sintomas de toxidez por metais pesados foram também constatados, experimentalmente, por Scharrer \& Schorpp (1936), Rehab \& Wallace (1978). Supõe-se, por outro lado, que mecanismos fitotóxicos de um íon metálico qualquer envolvem diferentes vias metabólicas em diferentes espécies ou variedades (Foy et al., 1978). No entanto, conforme aqui constatado, ambos os clones apresentaram aspectos de tolerância à presen ça de cobre, pois mostraram bom desenvolvimento em soluções de $10^{-6}, 10^{-5}$, $10^{-4}$ e $10^{-3} \mathrm{Mol} / 1$ de $\mathrm{CuSO}_{4}$, durante os três primeiros meses de observação. Evidenciou-se, porém, que nas plantas provenientes de Sanga Negra, após mais de um mês de exposição, os sintomas de toxidez foram mais precoces do que os constatados em plantas de Seival. Ao mesmo tempo que os sintomas tóxicos se acentuavam, manifestavam-se mecanismos de excreção de cristais de $\mathrm{CuSO}_{4}$ nas folhas, em concentrações mais altas do que $10^{-3} \mathrm{Mol} / \mathrm{l}$. Ernst (1974), referindo-se a ecótipos de solos metalf́feros, afima que Armeria maritima tem a capacidade de regular a quantidade de metais pesados em seus tecidos através da sua excreção. Essa espécie, quando submetida a soluções nutritivas com grandes quantidades de íons, formava gotículas sobre as folhas, que a baixa umidade do ar transformavam em sais que continham os fons da solução. $\mathrm{O}$ mesmo autor ainda afirma que esta reação é dependente da quantidade de sal existente no meio e que este mecanismo foi considerado por outros cientistas como um tipo de regulação.

As quantidades absorvidas pelas plantas submetidas a concentrações de $10^{-1} \mathrm{Mol} / 1$, cujos tecidos das folhas encontram-se praticamente mortos, demonstraram que o transporte, apesar de todas as alterações, continuava ativo. Os valores de $610 \mathrm{ppm}$ e $525 \mathrm{ppm}$ de cobre, constatados para as plantas expostas a soluções de $10^{-3} \mathrm{Mol} / 1$, demonstraram ser esta concentração o limite para o desenvolvimento das plantas, visto que, nestas concentrações, os sintomas de toxidez já foram evidentes, principalmente nas plantas de Sanga Negra. Comportamentos diferentes em clones de áreas de mineração, como os aqui constatados, já foram também observados por Hogan \& al. (1977b), Coughtrey \& al. (1978), Wainwrigh \& Woolhouse (1977) e Karataglis (1978).

\section{Conclusões}

As espécies encontradas em solos metal-tóxicos são muito variadas e diferem de acordo com o local, com as condições ecológicas e com a área geográfica. A seqüência das trocas gênicas, responsáveis pela colonização das áreas contaminadas por metais pesados, é, segundo Antonovic et al. (1971), a unidade recorde da seleção natural. Schinus lentiscifolius é uma destas espécies, apresentando dois clones com mecanismos de tolerância distintos à presença de cobre em seus tecidos. O clone da área mais rica em cobre (Seival) está adapta- 
do às mais altas concentrações, ao contrário do clone da área mais pobre em cobre (Sanga Negra). Ambas apresentam mecanismos de tolerância, como a excreção de excesso de íons através da superfície das folhas, após um valor-limite de acumulação de sais destes íons nos tecidos. Ao mesmo tempo, os mecanismos de resistência na germinação, em ambos os clones, são distintos em relação à taxa de germinação das sementes, a qual é menor (36\%) no clone que apresentou maior tolerância, (Seival). Isto pode ser explicado através da seleção sofrida por plantas destes ambientes, que expressam número reduzido de indivíduos, porém com maior potencial gênico para tolerar as concentrações altas de cobre no solo. Esta maior tolerância, além de ser comprovada pela maior resistência na germinação das sementes, também o é pelo aparecimento mais tardio de sintomas de toxidez e pela maior capacidade de acumulação do íon metálico testado nas plantas filhas.

\section{Referências Bibliografficas}

ANTONOVICS, J., BRADSHAW, A.B. \& TURNER, R.G. 1971. Heavy metal tolerance in plants. Adv. Ecol. Res. 7: 1-85.

BAUMEISTER, W. \& ERNST, W. 1978. Mineralstoffe und Pflanzenwachstum. Stuttgart, Gustav Fischer.

BROOKS, R.R. 1972. Geobotony and biogeochemistry in mineral exploration, New York, Harper and Row.

COUGHTREY, P.J. \& MARTIN, M.H. 1978. Cadmium uptake and distribution in tolerante and non-tolerante populations of Holcus lanatus grown in solution culture. $\mathrm{Oi}$ kos 30:555-560.

COUGRTREY, P.J., MARTIN, M.H. \& SHALES, S.W. 1978. Preliminary observations on cadmium tolerance in Holcus lanatus L. from soils artificially contaminated with heavy metals. Chemosphere 2: 193-198.

ERNST, W. 1974. Schwermetallvegetation der Erde. jStuttgart, Gustav Fischer.

1974. Physiology of heavy metal: resistance in plants. In: Symp. Proc. int. Conf. Heavy Metals Environ., Toronto 2 (1):121-136.

FOY, C.D., CHANEY, R.L. \& WHITE. M.C. 1978. The physiology of metal toxicity in plants. Ann. Rev. Plant. Physiol. 29:511-566.

GUPTA. B. \& MUKHERJI, S. 1977. effects of toxic concentrations of copper and growth and metabolism of rice seedlings. Z. Pfl. Physiol. 82:95-106.

HOGAN, G.D., COURTIN, G.M. \& RAUSER, W.E. 1977a. The effects of soil factors on the distribution of Agrostis gigantea on a mine wate site. Can. J. Bot. 55: 1038-1042.

HOGAN, G.D., COURTIN, G.M. \& RAUSER, W.E. 1977b. Copper tolerance in clones of Agrostis gigantea from a mine waste site. Can. J. Bot. 55: 1043-1050.

HORSCHRAFT, F.D.M. 1961. Vegetation. In: MENDELSON, F. The geology of the northern Rhodesian copperbelt. London.

HOWARD-WILLIAMS, C. 1971. Morphological variation between isolated populations of Becium homblei (De Wild) Duvig. et Plancke growing on heavy metal soils. Vegetatio 23 (2-4-): 141-151. 
HUNTER, J.G. \& VERGANO, O. 1953. Trace-elements toxities in oat plants. Ann. Appl. biol. 40: 461-777.

KARATAGLIS, S.S. 1978. Studies on heavy metal tolerance in populations of Anthoxanthum odoratum. Ber. Deutsch Bot. Ges. 91: 205-216.

KOTZ, L.M., KAISER, G., TSCHÖPEL, P. \& TÖLG, G. 1972. Aufschluss biologischer Matrices für die Bestimmung sehr niedriger Spurenselmentgehalte bei begrenzter einwaage mit Salpetersäure unter Druck in einem Teflongefäss. Anal. Chem. 260: 207-209.

LEINZ, V. \& PINAGEL, H. 1945. Estanho e tungstênio no Rio Grande do Sul. D.N.P.M., D.F.P.M., Bol. 70.

MILES, L.J. \& PARKER, G.R. 1979. The effects of soil-added cadmium on several plant species. J. Environ. Qual. 8 (2): 229-232.

MUKHERJI, S. \& DAS GUPTA B. 1972. Characterisation of Copper toxicity in lettuce seedlings. Physiol. Plant. 27: 1126-1929.

PORTO, M.L. 1981. Beitäge zur Schwermetallvegatition von Rio Grande do Sul Brasilien. Universidade de Ulm. Tese de doutorado.

PORTO, M.L. 1986. Vegetação metalófila e o desenvolvimento mineral. In: Anais do I Simpósio do Trópico Úmido, Belém, v. 2.

REHAB, F.I. \& WALLACE, A. 1978. Excess trace metal effects in cotton: 6. Nickel and cadmium in yellow loam soil. Commun. Soil. Sci. Plant. Anal. 9 (8): 779-784.

SCHARRER, K. \& SCHROPP, W. 1936. Über die Wirkung des Bleis auf das das Pflanzenwachstum. Z. Pflanzenernahr. Dug. Bodenk. 43: 34-42.

WAINWEIGHT, S.J. e WOOLHOUSE, H.W. 1977. Some physiological aspects of copper and zinc tolerance in Agrostis tenus Sibth: cell ellongation and membrane damage. J. Exp. Bot. 28 (5):1029-1036. 
Tolerância ao cobre em ecotipos de ...

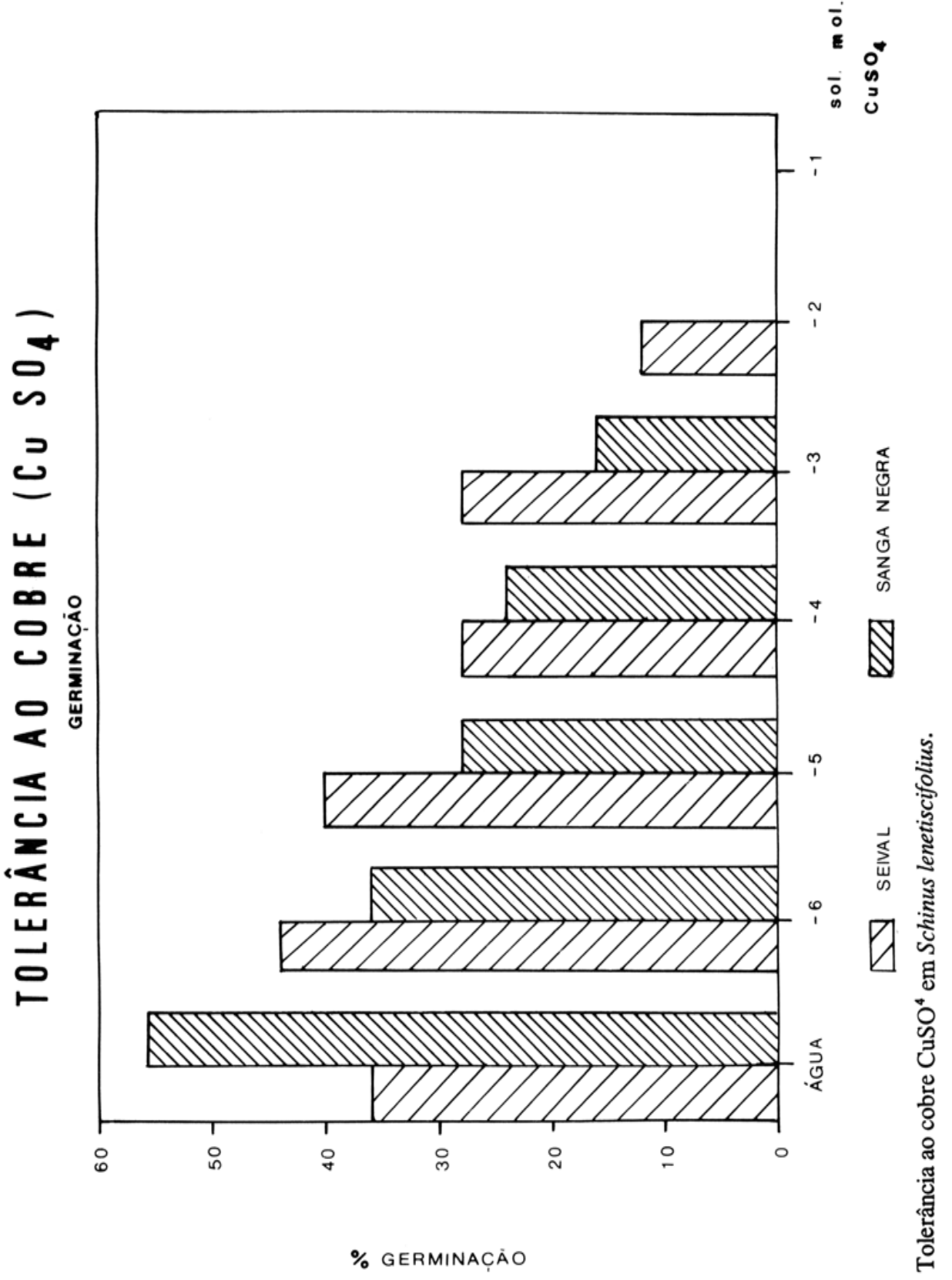

